\title{
A Hybrid Agent-Based Model of Chemotaxis
}

\author{
Zaiyi Guo and Joc Cing Tay \\ Evolutionary and Complex Systems Program \\ School of Computer Engineering, Nanyang Technological University, Nanyang Avenue, \\ Singapore 639798 \\ asjctay@ntu.edu.sg
}

\begin{abstract}
Models for systems biology commonly adopt Differential Equations or Agent-Based modeling approaches for simulating the processes as a whole. These choices need not necessarily be mutually exclusive. We propose a hybrid agent-based approach where biological cells are modeled as individuals (agents) while chemical molecules are kept as quantities. This hybridization in entity representation entails a combined modeling strategy with agent-based behavioral rules and differential equations, thereby balancing requirements of modeling extensibility with computational tractability. We demonstrate the efficacy of this approach with a realistic model of chemotaxis based on receptor kinetics involving an assay of $10^{3}$ cells and $1.2 \times 10^{6}$ molecules. The simulation is efficient and the results are agreeable with laboratory experiments.
\end{abstract}

Keywords: Hybrid Models, Multi-Agents, Chemotaxis, Receptor Kinetics.

\section{Introduction}

A large part of systems biology involves mathematical models constructed to understand the biological system (such as the human immune system) as a whole [1] from integrating knowledge obtained from laboratory experiments on sub-parts of the system. To simulate these system level dynamics efficiently, such models necessarily assume compartmentalized homogeneity and spatial independence [2, 3]. An alternative approach that has been gaining popularity are Multi-Agent (or MA) models, which explicitly model biological cells and molecules as individual agents [4-7]. As the real biological systems comprise millions to trillions of cells, MA models inevitably face issues of computational tractability and model scalability [8]. Despite these drawbacks, MA models are appealing for their advantages in phenomenological encoding of biological cell behaviors as agent rules [8]. Attempts have been made to overcome the computational tractability; such as choosing an intermediate level of abstraction [6] and applying the model to a distributed or parallel computational framework [9].

Though MA models generally deal with individuals instead of quantities, we believe that the DE modeling techniques for characterizing aggregated dynamics can be applied to MA models as well, giving rise to a hybrid modeling approach. In this paper, we propose a hybrid approach for designing MA system models where cells remain modeled as individual agents and molecules kept as quantities. This approach is motivated by the morphological distinction between cells and molecules, where 
molecules are much smaller (in the order of nanometers vs. cells in the order of micrometers), more abundant, have less complex behaviors and are well-characterized by physical laws (such as mass action law and Fick's diffusion law) as compared to cells. We demonstrate the efficacy of this approach with a model of chemotaxis. Our experimental design mimics the Under-Agarose Assay [10]: an in vitro experiment designed to study the chemotactic behavior of cells. Simulation results are found to be comparable with laboratory experiments.

\section{Integrating Agents and Quantities}

We consider the hybridization on two levels: globally in the environment, and locally with a cell (on its surface and within its body).

The environment is where biological cells and molecules reside and interact. The environment handles the storage and dissemination of spatial proximity information of cells and molecules. Consider the neutrophils (a type of white blood cell) that are attracted to the site of an injury as a result of communicated information in the form of inflammatory signals. The spread of these inflammatory signals, which is essentially based on molecular diffusion, is an environment level event. Together with this, another exogenous event involves blood flow (within the blood vessels) that forces the neutrophils and other related immune cells and molecules to recirculate in the human body. And then, upon entering the damaged tissue, their movement becomes more governed by the process of chemotaxis. Therefore, the environment is not merely a passive 'holder' to place the agents, but an entity with its own activities and special properties (e.g., blood flows). How agents behave is greatly influenced by its environment, hence the modeling of the environment is vital to agent-based system models of microbiological phenomena. We propose a three-layer environment, namely, 1) An Agent Holder Layer, where all agents (individuals) reside, 2) A Molecule Space Layer, where all molecule distributions are recorded, 3) A Flow Field Layer, where background flows, such as blood flows and lymph flows are modeled. Fig. 1a illustrates the three layers as composable two-dimensional planes.

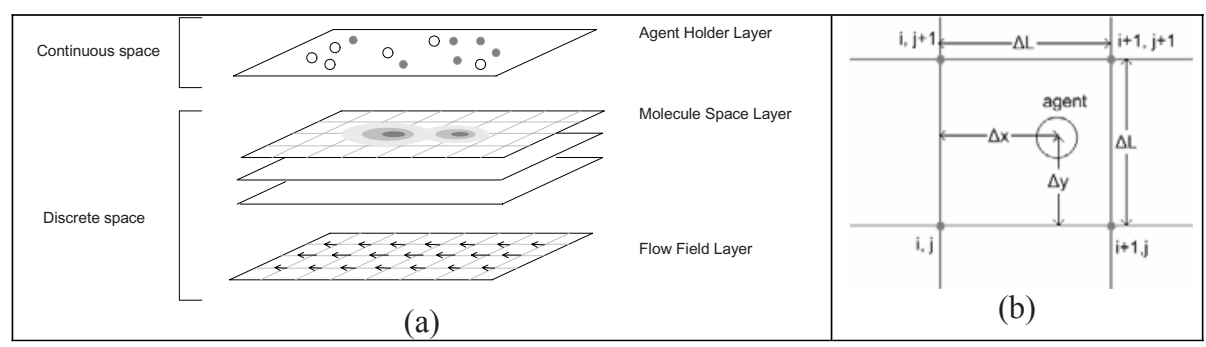

Fig. 1. (a) Three-layer structure of environment (b) Grid point interpolation by agent

The layers are either continuous or discrete in space. In continuous space layers, real-valued coordinates are used. In the discrete space layers, values are only stored at discrete 'grid points', hence values at real-valued coordinates may require 
interpolation. Of the three layers, only the Agent Holder Layer is continuous. The distance between two neighbouring grid points $\Delta L$, for a discrete space determines its granularity. Each grid point has a corresponding real-valued coordinate in the continuous space of the Agent Holder Layer, and each agent coordinate falls within a grid cell. The correspondence in spatial location enables spatial proximity-based interlayer communication.

The Molecule Space Layer consists of sub-layers, each representing the spatial distribution of a certain type of molecule. We let $Q_{i, j}$ denote the number of molecules surrounding the grid point $(i, j)$. In our model, the net change in molecule quantity over the entire spatial distribution is a combined result of 1) Molecular Production, where cells synthesize and release cytokines into its neighbourhood according to specific agent rules, 2) Molecular Diffusion, where molecules spread due to Brownian movement based on Fick's diffusion law, 3) Fluid Mechanics, in which molecules are 'pushed' by the local fluid flow within certain compartments such as the blood vessels and lymph duct. This is modeled by sampling the local velocity of the flow from the Flow Field Layer, and computing the quantity shift along the flow velocity by redistributing the molecular quantity to neighbouring grid points, and 4) Molecular Consumption / Degradation, where certain molecules are 'consumed' by binding to cell receptors. Molecules also decay through various pathways (e.g., instability or enzymic reactions), which is quantitatively specified by the decay rate constants $(\lambda)$ or half-life values $\left(t_{1 / 2}\right)$.

The Flow Field Layer characterizes the background flow in the environment. It represents the systematic transport superimposed onto the random movements of molecules and agents. The flow velocity $\mathbf{F}_{i, j}$ is specified for each grid point $(i, j)$, The Flow Field Layer is sampled by the Molecule Space Layer for computing the systematic shift of molecules, as stated in point (3) above.

The agents in the Agent Holder Layer move not only passively via systematic transport due to existence of flow, but also actively in response to the local molecular concentration and gradient, exhibiting chemokinesis and chemotaxis. Formally, we specify the displacement of an agent $\Delta \mathbf{p}$ within time $\Delta t$ as a function of the local flow velocity $\mathbf{F}_{\mathbf{p}}$, molecular concentration $C_{\mathbf{m}, \mathbf{p}}$ and gradient $\mathbf{G}_{\mathbf{m}, \mathbf{p}}$ for a set of molecules $\mathbf{m}$. Consider an agent in Fig. 1b, whose nearest grid points are $(i, j)$, $(i, j+1), \quad(i+1, j)$, and $(i+1, j+1)$. The local concentration of molecule $m$ at the agent's position obtained by bilinear interpolation is:

$$
\begin{gathered}
C_{m, \mathbf{p}}=\left(Q_{i, j} v_{x} v_{y}+Q_{i+1, j} w_{x} v_{y}+Q_{i, j+1} v_{x} w_{y}+Q_{i+1, j+1} w_{x} w_{y}\right) / \Delta L^{2} \\
\text { with } w_{x}=\Delta x / \Delta L, v_{x}=1-\Delta x / \Delta L, \quad w_{y}=\Delta y / \Delta L, v_{y}=1-\Delta y / \Delta L
\end{gathered}
$$

The local gradient $\mathbf{G}_{m, \mathbf{p}}=\nabla C_{m, \mathbf{p}}$ is a vector with components:

$$
\begin{aligned}
& G_{x}=\left(\left(Q_{i+1, j}-Q_{i, j}\right) v_{y}+\left(Q_{i+1, j+1}-Q_{i, j+1}\right) w_{y}\right) / \Delta L^{3} \\
& G_{y}=\left(\left(Q_{i, j+1}-Q_{i, j}\right) v_{x}+\left(Q_{i+1, j+1}-Q_{i+1, j}\right) w_{x}\right) / \Delta L^{3}
\end{aligned}
$$

The local flow velocity is:

$$
\mathbf{F}_{\mathbf{p}}=\mathbf{F}_{i, j} v_{x} v_{y}+\mathbf{F}_{i+1, j} w_{x} v_{y}+\mathbf{F}_{i, j+1} v_{x} w_{y}+\mathbf{F}_{i+1, j+1} w_{x} w_{y}
$$


The functional form of $\Delta \mathbf{p} / \Delta t$ is determined by the agent's ruleset based on its biological properties: some types of cells are highly motile and sensitive to molecular stimuli while others are rather fixed.

We have described the hybrid modeling of the environment. We next introduce the interaction rule building scheme for biological cells.

Biological cells are modeled at the individual level as agents, and their behaviors are specified by a set of rules. Most MA models use phenomenological if-then rules $[7,11]$. In reality, cell behaviors emerge from complex signaling pathways of molecular interactions and chemical reactions [6]. Therefore we believe the incorporation of molecular interactions (through surface receptors) into agent rules is necessary for a more quantitatively accurate MA model. As a first step, we develop a model for cellular locomotion through receptor kinetics [12]. We assume an agent's behaviour is governed by the following events: 1) Receptor-ligand binding, where free ligand molecules bind to receptors on the cell surface, 2) Receptor internalization and ligand consumption, where the receptor-ligand complex are internalized into the cytosol, and ligand is 'used up'/digested by other biological processes, and 3) Receptor recycling, where the previously internalized receptors are returned to the cell surface, available for binding.

Table 1. The individual reaction rules and corresponding quantity change equations

\begin{tabular}{|c|c|c|}
\hline Individual Reaction & Quantity Change & \\
\hline $\begin{array}{r}\text { Receptor-ligand binding } \\
\qquad L+R \stackrel{k b}{\longrightarrow} L R\end{array}$ & $\frac{d[L]}{d t}=-K_{b}[L][R]$ & (4) \\
\hline $\begin{array}{l}\text { Receptor internalization and ligand } \\
\text { consumption }\end{array}$ & $\frac{d[R]}{d t}=-K_{b}[L][R]-K_{r}\left[R^{*}\right]$ & (5) \\
\hline $\begin{array}{c}\qquad R \underset{k_{i}}{\longrightarrow} R^{*} \\
\text { Receptor recycling }\end{array}$ & $\frac{d[L R]}{d t}=K_{b}[L][R]-K_{i}[L R]$ & (6) \\
\hline$R * \stackrel{k_{r}}{\longrightarrow} R$ & $\frac{d\left[R^{*}\right]}{d t}=K_{i}[L R]-K_{r}\left[R^{*}\right]$ & (7) \\
\hline
\end{tabular}

The leftmost panel in Table 1 gives the molecular reactions of the three events underlying receptor kinetics. $L$ and $R$ denote individual ligand and receptor molecules respectively. The labels on the arrows characterize the probability of reaction per unit time. The right panel gives the corresponding differential equations, which are the macroscopic quantity-level changes that emerge from the individual reactions and are derived through mass action law. The rate constants $K_{x}$ $(x \in\{b, i, r\})$ are proportional to their microscopic counterpart $k_{x}$ and denote the number of reactions per unit time per reactant(s). By convention, [•] represents the concentration of the molecules in the whole space, but here we will define it as quantities of molecules in the proximity of, or within the agent. Readers may find these equations familiar, however, we emphasize that instead of a direct overall description of the molecular kinetics, these equations are local to an agent. 
Table 2. Agent Rule: Receptor-Ligand Interaction

\author{
If Alive Then \\ Sample the environment: get current local ligand quantity $[L]$ : \\ Get the values of individually stored quantities $[R],[L R]$, and $\left[R^{*}\right]$ \\ Compute $d[L], d[R], d[L R], d\left[R^{*}\right]$ using equations (4) - (7) \\ Update local quantities: $[X]=[X]+d[X]\left(X \in\left\{R, L, L R, R^{*}\right\}\right)$
}

End If

Table 2 expresses the agent rule that utilizes these equations for updating the local molecule quantities $[L],[R],[L R]$, and $[R *]$. The rule we specify here is quite flat as there is only one proposition and all the equations appear in the action part. However, it is possible to specify more complex situations that an agent can be in and the different responses it could make. We incorporate this receptor-ligand interaction in a hybrid model of chemotaxis in the next Section.

\title{
3 Chemotaxis
}

Chemotaxis refers to the directed cell movement towards or away from a chemical source (chemoattractant or chemorepellent) by sensing and following the chemical gradient [13]. Through chemotaxis, a sperm cell finds the ovum, and a white blood cell finds the place of injury or inflammation [13]. Much of the research effort has been devoted to discover the mechanisms that transduce the extracellular chemical signals into cell locomotion [14-16], including molecular events such as G-protein activation, lipid remodeling, protein kinase activation, calcium elevation $[13,15]$ and cellular events such as cell polarization, pseudopod extension, excitation and adaptation [13, 16]. However, these molecular details are seldom captured quantitatively into models for simulation. Keller and Segel used Partial-DEs to model chemotaxis [17]: both cells and molecules are treated as quantities, hence the intracellular molecule kinetics are largely ignored. Our model differs in that chemotactic behaviors are described at an individual level instead of a population level. Though the model we present here is simplified, the hybrid approach makes it possible to incorporate more complex intracellular signaling pathways for future extensions. We next describe how an agent's displacement is computed based on our model of chemotaxis.

We define the overall displacement of an agent (or cell) as the vector sum of Brownian, chemotactic and systematic displacement due to external fluid flows. In this experiment, we define chemotactic response to be proportional to the difference in the receptor occupancy of the front and rear of the cell [18]. The receptor-ligand interactions have been described in Equation (4) to (7). Let $r$ and $V$ represent the radius and the volume of an cell, and let $[R],[L R],\left[R^{*}\right]$ represent the quantities of the free surface receptors, occupied surface receptors and the internalized receptors respectively. The amount of ligand available at the front and the rear of the cell is approximated by: 


$$
[L]_{\text {front }}=(C+|\mathbf{G}| r) V / 2,[L]_{\text {rear }}=(C-|\mathbf{G}| r) V / 2
$$

The change in newly bound receptors $(L R)$ in time $\Delta t$ at the front and rear are:

$$
\Delta[L R]_{\text {front }}=K_{b}[L]_{\text {front }}[R]_{\text {front }} \Delta t, \Delta[L R]_{\text {rear }}=K_{b}[L]_{\text {rear }}[R]_{\text {rear }} \Delta t
$$

and we assume $[R]_{\text {front }}=[R]_{\text {rear }}=[R] / 2$. The difference in newly bound receptors between the cell front and the rear in time $\Delta t$ is therefore

$$
\Delta[L R]_{\text {diff }}=K_{b}\left([L]_{\text {front }}-[L]_{\text {rear }}\right)[R] \Delta t / 2
$$

Assuming linear dependency of the agent's displacement on $\Delta[L R]_{\text {diff }}$ gives rise to

$$
\Delta \mathbf{p}=k \Delta[L R]_{\text {diff }} \mathbf{G} /|\mathbf{G}|
$$

where $k$ is a proportionality parameter. The larger the value of $k$, the more sensitive is the cell is to the change in receptor occupancy at its front and rear.

We conduct an in silico simulation of the Under-Agarose Assay, which was designed to investigate the in vitro chemotactic behavior of lymphoblastic cells [10]. Fig. 2 illustrates the assay and a sample result. The tissue culture dish is filled with Agarose (gel) and two wells are incised, where one is filled with cells and the other with a chemoattractant (lysophosphatidylcholine). The cells are observed to migrate towards the chemoattractant well by squeezing under the gel (Fig. 2).

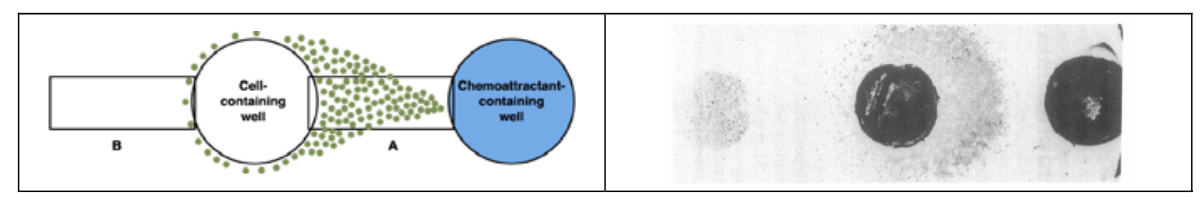

Fig. 2. Under-Agarose Assay (Figures adopted from [10] and [19])

Table 3. Model Parameters for Chemotaxis Experiment

\begin{tabular}{lll}
\hline Parameter & Value & Description \\
\hline$\Delta t$ & $1 \mathrm{~min}$ & Time-step \\
\hline$\Delta L$ & $20 \mu \mathrm{m}$ & Granularity of the site grid \\
\hline$D$ & $10 \mu \mathrm{m}^{2} \mathrm{~min}^{-1}$ & Diffusion constant of molecule $L$ \\
\hline$V$ & $50 \mu \mathrm{m}^{2}$ & Volume of cell \\
\hline$r$ & $4 \mu \mathrm{m}$ & Radius of cell \\
\hline$l$ & $2 \mu \mathrm{m} \mathrm{min}$ & Random motility of cell: max step length per unit time \\
\hline$R_{\text {tot }}$ & 50000 & The total amount of receptor molecule $R$ per cell \\
\hline$K_{r}$ & $0.5 \mathrm{~min}^{-1}$ & The recycle rate of internalized receptor $R^{*}$ \\
\hline$K_{b}$ & $2 \times 10^{-6} \mathrm{~min}^{-1}$ & The association rate of $L$ and $R$ \\
\hline$K_{i}$ & $0.3 \mathrm{~min}^{-1}$ & The internalization rate of occupied receptor $L R$ \\
\hline
\end{tabular}

To mimic the Under-Agarose Assay, we construct a virtual environment as a $3 \mathrm{~mm}$ by $2 \mathrm{~mm}$ enclosed two-dimensional space (Fig. 3, left). The cell and attractant wells are both $250 \mu \mathrm{m}$ in radius and are placed $1 \mathrm{~mm}$ apart (Fig. 3, left, red circle). Initially, 
the cell well is filled with 1000 cells, and the attractant well is filled with attractant molecules at a concentration of 2500 particles per $20 \mu \mathrm{m} \times 20 \mu \mathrm{m}$ area (for a total of about $1.2 \times 10^{6}$ molecules). Table 3 summarizes the parameter values for the simulation.

The simulation model is implemented in Java. The simulation is run on a Windows XP Professional system with $2.4 \mathrm{GHz}$ Pentium $4 \mathrm{CPU}$ and $1 \mathrm{~GB}$ of Memory. One single run of the simulation takes approximately 3 minutes. The video capture of the simulation can be downloaded freely from http://www.ntu.edu.sg/ home/asjctay/data/chemotaxis.zip (7.56 MB in size).

Fig. 3 shows the simulation results with $k$ set to 5 and 30 . The left figures show the migration pattern at time step $\mathrm{t}=5000$. Both exhibit the biased random walk of the cells towards the attractant, and with larger $k$ values, the spread accelerates. Due to ligand consumption, the space occupied by the cells is at a lower concentration, resulting in a sharp gradient at the edge of the diffusing molecular population, which promotes the movement of cells. The right figures show the movement of the front $\left(x_{\max }\right)$, rear $\left(x_{\min }\right)$, and mean $\left(x_{a v g}\right)$ positions of the molecular migration shape over time. $L_{x}$ and $L_{y}$ denotes the bounding width and length of this shape along the $\mathrm{x}$ and y axes, and $L_{x} / L_{y}$ measures its distortion. Interestingly, though the spread towards the source of the attractant is evident, the average shift along the $\mathrm{x}$ axis is very small,

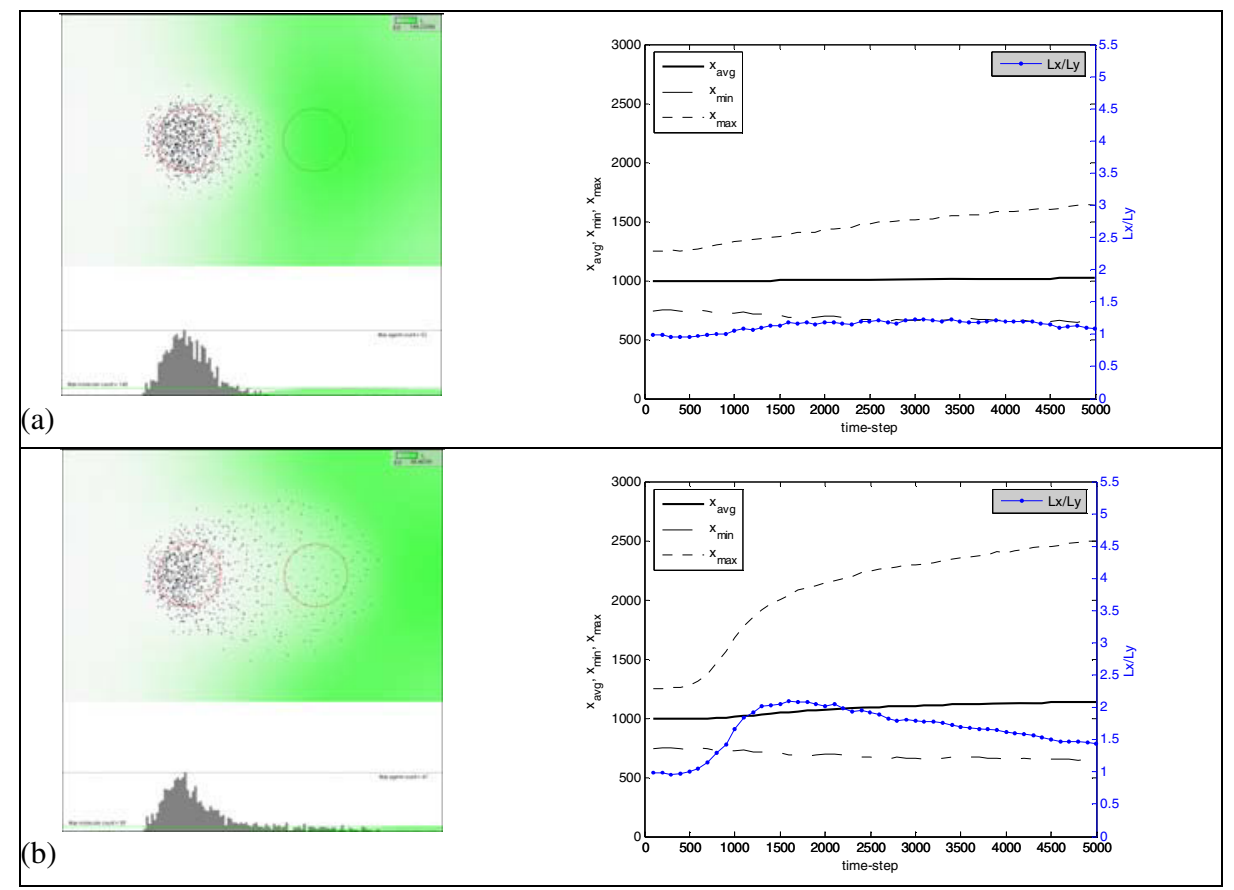

Fig. 3. Simulation results of Chemotaxis at time steps (a) $k=5$ and (b) $k=30$ 
as a large amount of the cells are still within the cell well and exhibit no chemotactic movement. This is likely due to low concentration created by the front-end cells, hence the rear-end cells are blocked from sensing the distant gradient. For $k=30$ the distortion maximizes at $\mathrm{t}=1500$, showing that cells are attracted along the $\mathrm{x}$ axis. The distortion then slowly decreases, indicating increasing spread along the $y$ axis due to gradient created at the edge of the migration shape. The cells spread rather than move with a narrow front, hence our results differ from the illustration from [10] but closer to the photo in [19] (see Fig. 2). Our simulation results show that the spread is caused by the distortion of the gradient landscape due to both diffusion and interactions between cells and molecules. The efficacy of the hybrid approach to construct agentbased models for biological phenomena has been demonstrated with a model of chemotaxis verified qualitatively with laboratory experimentation.

\section{Conclusions}

We present a hybrid agent-based modeling approach, where biological cells are modeled as individual agents and molecules as quantities, thereby balancing requirements of modeling extensibility (i.e. to handle complex cell behaviors) with computational tractability. The hybridization of the quantity-based macroscopic modeling (for molecules) and the individual-based microscopic modeling (for cells) exists at both the environment and individual levels. The environment consists of three layers: an Agent Holder Layer, a Molecule Space Layer (divided into several sub-layers), and a Field Layer. Agents (individual cells) interact with molecules (quantities) based on spatial proximity. The model allows agent behaviors to be specified by differential equations, if-then logic, or a hybrid of both. The result is a modeling approach that provides extensibility and increased accuracy based on available information. We demonstrate the efficacy of this hybrid approach with a realistic model of chemotaxis based on receptor kinetics for an Under-Agarose assay of $10^{3}$ cells and $1.2 \times 10^{6}$ molecules. The experiment successfully and efficiently reproduces a "biased random walk" as reported in the research literature, with results showing strong agreement with migration patterns observed from laboratory experiments.

\section{References}

1. Kitano, H.: System Biology: A Brief Overview. Science 295 (2002) 1662-1664

2. Perelson, A.S., Nelson, P.W.: Mathematical Analysis of HIV-1 Dynamics in Vivo. SIAM Review 41 (1999) 3-44

3. Stekel, D.J., Parker, C.E., Nowak, M.A.: A Model of Lymphocyte Recirculation. Immunology Today 18 (1997) 216-221

4. Casal, A., Sumen, C., Reddy, T.E., Alber, M.S., Lee, P.P.: Agent-Based Modeling of the Context Dependency in T Cell Recognition. Journal of Theoretical Biology 236 (2005) 376-391

5. Webb, K., White, T. Cell Modeling Using Agent-Based Formalisms. In: AAMAS'04, New York, USA (2004) 
6. Khan, S., Makkena, R., McGeary, F., Decker, K., Grills, W., Schmidt, C. A Multi-Agent System for the Quantitative Simulation of Biological Networks. In: AAMAS 03, Melbourne, Australia (2003) 385-392

7. Guo, Z., Han, H.K., Tay, J.C. Sufficiency Verification of HIV-1 Pathogenesis Based on Multi-Agent Simulation. In: Proceedings of the Genetic and Evolutionary Conference 2005, Vol. I. Washington D.C. (2005) 305-312

8. Guo, Z., Tay, J.C. A Comparative Study of Modeling Strategies of Immune System Dynamics under HIV-1 Infection. Lecture Notes in Computer Science, Vol. 3627. Springer-Verlag, Banff, Albeta, Canada (2005) 220-233

9. Logan, B., Theodoropoulos, G.: The Distributed Simulation of Multi-Agent Systems. Proceedings of the IEEE 89 (2001) 174-185

10. Heit, B., Kubes, P.: Measuring Chemotaxis and Chemokinesis: The under-Agarose Cell Migration Assay. Sci. STKE 170 (2003) pl5.

11. Jacob, C., Litorco, J., Lee, L. Immunity through Swarms: Agent-Based Simulations of the Human Immune System. In: Proceedings of 3rd International Conference on Artificial Immune Systems, Vol. 3239. Springer-Verlag GmbH, Catania, Sicily, Italy (2004) 400-412

12. Zigmond, S.H., Sullivan, J.L., Lauffenburger, D.A.: Kinetic Analysis of Chemotactic Peptide Receptor Modulation. The Journal of Cell Biology 92 (1982) 34-43

13. Eisenbach, M., Lengeler, J.W., Varon, M., Gutnick, D., Meili, R., Firtel, R.A., Segall, J., E., Omann, G.M., Tamada, A., Murakami, F.: Chemotaxis. Imperial College Press, London (2004).

14. Mitchison, T.J., Cramer, L.P.: Actin-Based Cell Motility and Cell Locomotion. Cell 84 (1996) 371-379

15. Firtel, R.A., Chung, C.Y.: The Molecular Genetics of Chemotaxis: Sensing and Responding to Chemoattractant Gradients. BioEssays 22 (2000) 603-615

16. Sanchez-Madrid, F., Del Pozo, M.A.: Leukocyte Polarization in Cell Migration and Immune Interactions. The EMBO Journal 18 (1999) 501-511

17. Keller, E.F., Segel, L.A.: Model for Chemotaxis. Journal of Theoretical Biology 30 (1971) 225-234

18. Devreotes, P.N., Zigmond, S.H.: Chemotaxis in Eukaryotic Cells: A Focus on Leukocytes and Dictyostelium. Ann. Rev. Cell. Biol. 4 (1988) 649-686

19. Hoffman, R.D., Klingerman, M., Sundt, T.M., Anderson, N.D., Shin, H.S.: Stereospecific Chemoattraction of Lymphoblastic Cells by Gradients of Lysophosphatidylcholine. Proc. Natl. Acad. Sci. USA 79 (1982) 3285-3289 